\title{
Rationalizability and Nash equilibria in guessing games
}

Citation for published version (APA):

Seel, C., \& Tsakas, E. (2017). Rationalizability and Nash equilibria in guessing games. Games and Economic Behavior, 106, 75-88. https://doi.org/10.1016/j.geb.2017.09.013

Document status and date:

Published: 01/11/2017

DOI:

10.1016/j.geb.2017.09.013

Document Version:

Publisher's PDF, also known as Version of record

Document license:

Taverne

Please check the document version of this publication:

- A submitted manuscript is the version of the article upon submission and before peer-review. There can be important differences between the submitted version and the official published version of record.

People interested in the research are advised to contact the author for the final version of the publication, or visit the DOI to the publisher's website.

- The final author version and the galley proof are versions of the publication after peer review.

- The final published version features the final layout of the paper including the volume, issue and page numbers.

Link to publication

\footnotetext{
General rights rights.

- You may freely distribute the URL identifying the publication in the public portal. please follow below link for the End User Agreement:

www.umlib.nl/taverne-license

Take down policy

If you believe that this document breaches copyright please contact us at:

repository@maastrichtuniversity.nl

providing details and we will investigate your claim.
}

Copyright and moral rights for the publications made accessible in the public portal are retained by the authors and/or other copyright owners and it is a condition of accessing publications that users recognise and abide by the legal requirements associated with these

- Users may download and print one copy of any publication from the public portal for the purpose of private study or research.

- You may not further distribute the material or use it for any profit-making activity or commercial gain

If the publication is distributed under the terms of Article $25 \mathrm{fa}$ of the Dutch Copyright Act, indicated by the "Taverne" license above, 
Note

\title{
Rationalizability and Nash equilibria in guessing games
}

\author{
Christian Seel*, Elias Tsakas* \\ Department of Economics (AE1), Maastricht University, P.O. Box 616, 6200 MD, Maastricht, The Netherlands
}

\section{A R T I C L E I N F O}

\section{Article history:}

Received 13 March 2017

Available online 6 October 2017

\section{JEL classification:}

C70

$\mathrm{C} 72$

Keywords:

Guessing game

Beauty contest

Rationalizability

\begin{abstract}
A B S T R A C T
Games in which players aim to guess a fraction or multiple $p$ of the average guess are known as guessing games or ( $p$-)beauty contests. In this note, we derive a full characterization of the set of rationalizable strategies and the set of pure strategy Nash equilibria for such games as a function of the parameter $p$, the number of players and the (discrete) set of available guesses to each player.
\end{abstract}

(C) 2017 Elsevier Inc. All rights reserved.

\section{Introduction}

In guessing games - also called ( $p$-)beauty contests - each player chooses a number and the player whose number is closest to $p$ times the average of all numbers wins a fixed prize. Such a game with $p=\frac{2}{3}$ was first used in Ledoux (1981) and brought to the attention of an economic audience by Moulin (1986). Since the seminal paper by Nagel (1995), guessing games have attracted a lot of interest among behavioral and experimental economists. Moreover, they have become the leading example for teaching iterative reasoning processes in game theory courses.

The popularity of this class of games results - at least partly - from the gap between empirical evidence and "theoretical predictions". Roughly speaking, these predictions are typically based on the following informal reasoning: for $p=\frac{2}{3}$, two thirds of the average is further from the highest than from the second highest number irrespective of what the opponents choose. Therefore, the highest number will never be chosen. By iteratively applying the same reasoning, all numbers except the smallest one are eliminated. ${ }^{1} \mathrm{~A}$ similar argument suggests that for $p>1$, only the highest number should be chosen.

In this note, we formalize the "theoretical predictions" using the two standard solution concepts, viz., rationalizability and Nash equilibrium. Rationalizability imposes two basic assumptions, namely that every player chooses a strategy that is optimal given his belief about the opponents strategies (rationality) and it is transparent across the players that every player is rational (common belief in rationality). Nash equilibrium additionally requires each player's beliefs to be correct.

\footnotetext{
We are indebted to Sebastian Ebert, an Associate Editor and two anonymous referees for valuable comments. Elias thanks the Department of Economics at UC Davis for its hospitality while working on this project. This paper supersedes an earlier draft titled "Rationalizability in Guessing Games".

* Corresponding authors.

E-mail addresses: c.seel@maastrichtuniversity.nl (C. Seel), e.tsakas@maastrichtuniversity.nl (E. Tsakas).

URLs: https://www.maastrichtuniversity.nl/c.seel (C. Seel), http://www.elias-tsakas.com/home.html (E. Tsakas).

1 Experimental findings are usually at odds with these predictions. This discrepancy is typically attributed to limited depth of reasoning and different behavioral models try to capture it, e.g., the level-k model (Ho et al., 1998; Bosch-Doménech et al., 2002) or the cognitive hierarchy model (Camerer et al., 2004).
} 
For the general case, where the set of possible guesses is an arbitrary finite set of non-negative real numbers, we first show that all (pure-strategy) Nash equilibria are symmetric. Moreover, we characterize the set of parameters $p$ for which an arbitrary symmetric strategy profile is a Nash equilibrium. Our analysis generalizes the one of López (2001), who restricts attention to parameters $p<1$ and integer guesses. Then, we characterize the set of rationalizable strategies as the set of guesses that lie between the "lowest" and the "highest" symmetric Nash equilibrium. For a large range of parameters, all guesses are rationalizable, i.e., there are no stupid guesses.

Subsequently, we focus on a special case that frequently appears in experiments, where the set of possible guesses consists of consecutive nonnegative integers. For $p \leq 1$, a strategy is rationalizable if and only if it is part of a symmetric pure-strategy Nash equilibrium. For $p \geq 1$, there is a threshold parameter (equal to half of the number of players), such that only the highest guess is rationalizable for $p$ above the threshold, but every guess is rationalizable otherwise.

This note does not intend to explain the differences between theoretical predictions and empirical findings. Instead, it provides an in-depth game-theoretic analysis for one of the most famous games in the literature. Moreover, it emphasizes that the aforementioned informal treatment is inappropriate for computing standard theoretical predictions and that caution in experimental design and (undergraduate) teaching is asked for.

We proceed as follows. Section 2 sets up the general model and derives the main results. Readers who are familiar with the model and only interested in the predictions for the most frequently analyzed case in experiments can skip to Section 3. A sketch of the proofs is presented in Section 4. In Section 5, we discuss extensions of the model. The details of the proofs are presented in the Appendix.

\section{Setting and main results}

Consider a (symmetric) normal form game with $I=\{1, \ldots, N\}$ being the set of players, where $N \geq 2$. For each player $i \in I$, the set of pure strategies (guesses) is denoted by $S_{i}=\left\{a_{0}, a_{1}, \ldots, a_{M}\right\}$ with $0 \leq a_{0}<a_{1}<\cdots<a_{M}<\infty$ and $M \geq 1$. A typical element of $S_{i}$ is denoted by $s_{i}$. For a given pure strategy profile $\left(s_{1}, \ldots, s_{N}\right)$, the target guess is defined as $\frac{p}{N} \sum_{j \in I} s_{j}$, where $p \in \mathbb{R}_{+}$is an exogenous parameter. Players compete for a fixed prize. The player whose guess is closest to the target guess wins the prize; ties are broken randomly with equal probability. All players are assumed to be expected utility maximizers.

If $N=2$, it is straightforward that for $p<1$ (resp., for $p>1$ ) the only Nash equilibrium is the pure strategy profile $\left(a_{0}, a_{0}\right)$ (resp., the pure strategy profile $\left(a_{M}, a_{M}\right)$ ), whereas for $p=1$ every strategy profile is a Nash equilibrium. Thus, we henceforth focus on games with at least three players. To characterize the set of Nash equilibria, we introduce some parameter thresholds. In particular, let

$$
p_{m}:=\frac{N}{2} \cdot \frac{a_{m-1}+a_{m}}{a_{m-1}+(N-1) a_{m}}
$$

for each $m \in\{1, \ldots, M\}$, with the convention $p_{0}:=0$. Moreover, define

$$
q_{m}:=\frac{N}{2} \cdot \frac{a_{m+1}+a_{m}}{a_{m+1}+(N-1) a_{m}}
$$

for every $m \in\{0, \ldots, M-1\}$, with the convention $q_{M}:=\infty$. Note that for $N>2$, we have $p_{m}<1<q_{m}$ for every $m \in$ $\{0,1, \ldots, M\}^{2}$

Theorem 1 (Characterization of Nash equilibria). Every (pure-strategy) Nash equilibrium is symmetric. Moreover, the following statements are true for every $m \in\{0, \ldots, M\}$ :

(i) For $p \leq 1$, the strategy profile $\left(a_{m}, \ldots, a_{m}\right)$ is a Nash equilibrium if and only if $p \geq p_{m}$.

(ii) For $p \geq 1$, the strategy profile $\left(a_{m}, \ldots, a_{m}\right)$ is a Nash equilibrium if and only if $p \leq q_{m}$.

Remark 1. By our conventions $p_{0}=0$ and $q_{M}=\infty$, it follows directly that $\left(a_{0}, \ldots, a_{0}\right)$ is a Nash equilibrium for every $p \leq 1$ and $\left(a_{M}, \ldots, a_{M}\right)$ is a Nash equilibrium for every $p \geq 1$.

We now turn attention to rationalizability. For each player $i \in I$, let $R_{i}^{0}:=S_{i}$ and inductively define the strategies $R_{i}^{k} \subseteq S_{i}$ that survive $k$ rounds of elimination of strictly dominated strategies (for a formal definition of $R_{i}^{k}$, see the appendix). Then, $R_{i}^{*}:=\bigcap_{k=0}^{\infty} R_{i}^{k}$ contains the (correlated) rationalizable strategies. These are exactly the strategies that can be played under rationality and common belief in rationality (Brandenburger and Dekel, 1987; Tan and Werlang, 1988). ${ }^{3}$

\footnotetext{
2 This follows from $p_{m}$ (resp., $q_{m}$ ) being strictly decreasing (resp., strictly increasing) in $N$, combined with the fact that $p_{m}=q_{m}=1$ when $N=2$.

3 For the rest of the paper, the term rationalizability refers to the concept of correlated rationalizability. In general, correlated rationalizability is a coarsening of Bernheim's (1984) and Pearce's (1984) (independent) rationalizability. In Section 5.1, we show that both rationalizability concepts yield exactly the same predictions for our model.
} 


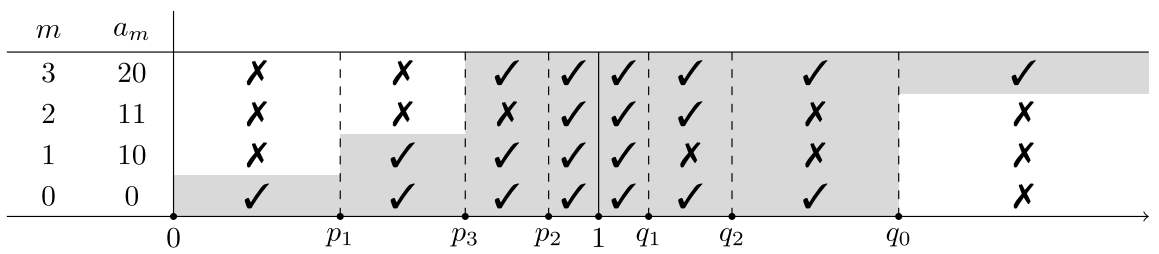

Fig. 1. Rationalizable strategies and Nash equilibria for $N=3, M=3, S_{i}=\{0,10,11,20\}$ and varying $p$. The symbol $\checkmark$ (resp., $\boldsymbol{x}$ ) signifies that the corresponding symmetric pure-strategy profile is (resp., is not) a Nash equilibrium for parameters $p$ within the interval. The shaded area consists of all parameters such the respective strategy is rationalizable.

Theorem 2 (Characterization of rationalizable strategies). A strategy $s_{i} \in S_{i}$ is rationalizable if and only if there exist symmetric pure-strategy Nash equilibria, $\left(a_{m}, \ldots, a_{m}\right)$ and $\left(a_{n}, \ldots, a_{n}\right)$, such that $a_{m} \leq s_{i} \leq a_{n}$. In particular, the following statements hold:

(i) For $p \leq 1$, the rationalizable strategies are $\left\{a_{0}, \ldots, a_{\bar{m}}\right\}$ with $\bar{m}=\max \left\{\ell \in\{0, \ldots, M\}: p \geq p_{\ell}\right\}$.

(ii) For $p \geq 1$, the rationalizable strategies are $\left\{a_{\underline{m}}, \ldots, a_{M}\right\}$ with $\underline{m}=\min \left\{\ell \in\{0, \ldots, M\}: p \leq q_{\ell}\right\}$.

Remark 2. Since $p_{0}=0$ and $q_{M}=\infty$, both $\left\{\ell \in\{0, \ldots, M\}: p \geq p_{\ell}\right\}$ and $\left\{\ell \in\{0, \ldots, M\}: p \leq q_{\ell}\right\}$ are nonempty and finite sets. Thus, they attain a maximum and a minimum respectively.

The following example provides an illustration of the main insights of Theorems 1 and 2.

Example 1. Let $N=3, M=3$ and $S_{i}=\{1,10,11,20\}$. We obtain $\left(p_{1}, p_{2}, p_{3}\right)=\left(\frac{11}{14}, \frac{63}{64}, \frac{31}{34}\right)$ and $\left(q_{0}, q_{1}, q_{2}\right)=\left(\frac{11}{8}, \frac{63}{62}, \frac{31}{28}\right)$, i.e., we have $p_{2}>p_{3}>p_{1}$ and $q_{1}<q_{2}<q_{0}$. Thus we can partition the set of values $p \in \mathbb{R}_{+}$into intervals and use our theorems to obtain the rationalizable strategies and the Nash equilibria within each of these intervals, as shown in Fig. 1.

Note that the set of Nash equilibria is not always "connected". For instance, when $p \in\left(p_{3}, p_{2}\right)$, the profiles $(20,20,20)$ and $(10,10,10)$ are both Nash equilibria, but $(11,11,11)$ is not. Intuitively, since $p_{m}$ and $q_{m}$ are not always monotonic in $m$, the conditions of Theorem 1 are sometimes satisfied for extreme values of $m$ and not for intermediate ones.

Unlike the set of Nash equilibria, the set of rationalizable strategies is always "connected". More precisely, by Theorem 2, the set of rationalizable strategies are those between the "lowest" and the "highest" Nash equilibrium. As $p_{m}$ and $q_{m}$ are not always monotonic in $m$, however, the set of rationalizable strategies may "jump" while $p$ increases. In our example, for $p<p_{3}$ the set of rationalizable strategies is $\{0,10\}$, whereas for $p \geq p_{3}$ the set of rationalizable strategies becomes $\{0,10,11,20\}$.

\section{The famous special case}

In this section, we consider the special case with a strategy set $S_{i}=\{0,1, \ldots, M\}$ with typical element $m$, which has been extensively studied in the experimental literature (e.g., see Ledoux, 1981; Nagel, 1995) and is often presented in textbooks.

Define two thresholds $p_{m}=\frac{2 N m-N}{2 N m-2}$ and $q_{m}=\frac{2 N m+N}{2 N m+2}$, maintaining the convention that $p_{0}=0$ and $q_{M}=\infty$. Theorem 2 from Section 2 reduces to the following corollaries:

Corollary 1. For $p \leq 1$, a strategy $m \in\{0, \ldots, M\}$ is rationalizable if and only if $p \geq p_{m}$.

Corollary 2. For $p \geq 1$, the following statements hold:

(i) All strategies $m \in\{0, \ldots, M\}$ are rationalizable if $p \leq \frac{N}{2}$.

(ii) Only the strategy $m=M$ is rationalizable if $p>\frac{N}{2}$.

We can decompose the parameter space into three regions (see also Fig. 2). In Region $1\left(p<p_{M}\right)$, more guesses become rationalizable as $p$ increases. Region $2\left[p_{M}, \frac{N}{2}\right]$ is the "experimenter's nightmare", viz., the region of no stupid guesses, where all strategies are rationalizable. Finally, in Region $3\left(p>\frac{N}{2}\right)$, only the highest guess is rationalizable. The parametrization in the original implementation by Ledoux (1981), where $p=\frac{2}{3}$ and $N=2898$, falls in Region 1 and only strategies $m \in$ $\{0,1\}$ are rationalizable. The treatment in the seminal paper by Nagel (1995), where $p=\frac{4}{3}$ and $N \in\{15,16,17,18\}$ falls in Region 2, i.e., all guesses are rationalizable.

The following example discusses the relation of rationalizable strategies and Nash equilibria in the general case versus the special case in more detail.

Example 2. As is often assumed in experiments, let $M=100$. The Nash equilibria and the rationalizable strategies are illustrated in Fig. 2 in a similar way as in Fig. 1 above. 


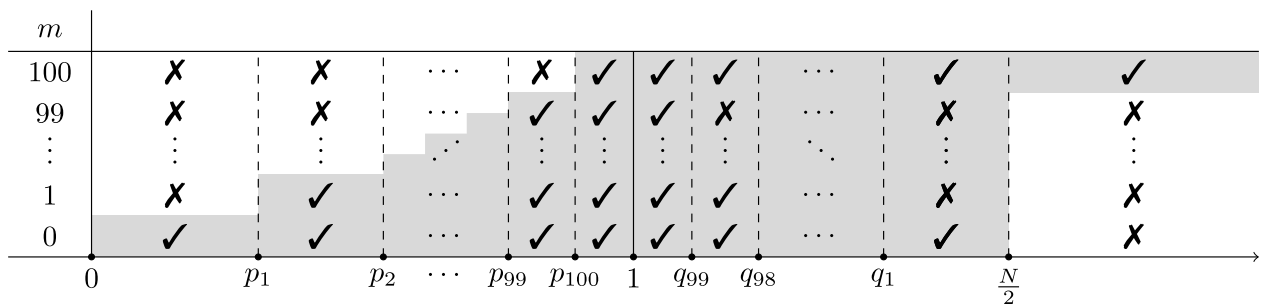

Fig. 2. Rationalizable strategies and Nash equilibria for $M=100$, i.e., $S_{i}=\{0,1, \ldots, 100\}$ and varying $p$. The symbol $\checkmark$ (resp., $\boldsymbol{x}$ ) signifies that the corresponding symmetric pure-strategy profile is (resp., is not) a Nash equilibrium for parameters $p$ within the interval. The shaded area consists of all parameters such the respective strategy is rationalizable.

For $p<1$, unlike in the general case, the set of Nash equilibria is "connected" and it coincides with the set of rationalizable strategies. This is why the condition $p \geq p_{m}$ - used to characterize the set of Nash equilibria in Theorem 1 (i) is also used here to identify the set of rationalizable strategies (Corollary 1 ). Furthermore, the fact that $p_{m}$ is monotonic in $m$ guarantees that for every $m \in\{0, \ldots, 100\}$ there is some $p \in(0,1)$ such that the set of rationalizable strategies is $R_{i}^{*}=\{0, \ldots, m\}$, i.e., there are no jumps of the highest rationalizable strategy as $p$ increases, unlike in Example 1 .

For $p>\frac{N}{2}$, we have $p>q_{m}$ for every $m \in\{0, \ldots, 99\}$. Thus, no strategy profile $(m, \ldots, m)$ with $m<100$ is a Nash equilibrium by Theorem 1 (ii) and hence no $m \in\{0, \ldots, 99\}$ is rationalizable. That is, if $m=0$ is eliminated then every strategy below $M=100$ will eventually be eliminated. On the other hand, if $p \in\left[1, \frac{N}{2}\right]$, the lowest strategy $m=0$ is not eliminated, and the unraveling does not begin. Finally, note that even in the special case, for some $p>1$, the set of Nash equilibria is "disconnected".

\section{Sketch of the proofs}

We split each proof into some intermediate steps, summarized in Lemmas 1-2 for Theorem 1 and Lemmas 3-4 for Theorem 2. This section only presents the general idea behind the proofs. The complete arguments are relegated to the appendix.

\subsection{Theorem 1: Nash equilibria}

Symmetry: Suppose there are at most two different guesses (say $a_{m}$ and $a_{n}$ ) chosen in equilibrium and that each player wins with positive probability. Since there are at least three players, one of the two guesses (say $a_{n}$ ) is picked by at least two players (say $i$ and $j$ ). Now suppose that $i$ switches to $a_{m}$. In this case, the target guess moves closer to $a_{m}$, meaning that $j$ is not among the possible winners anymore and thus the probability of $i$ winning increases. Hence, the original strategy profile (with both $a_{m}$ and $a_{n}$ chosen by some players) is not a Nash equilibrium. Thus, only symmetric strategy profiles can be Nash equilibria.

Let us proceed with Parts (i) and (ii) of the theorem. For notation simplicity, let $\bar{a}_{m}$ denote the strategy profile where all players choose $a_{m}$ and let $\bar{a}_{m}^{-i}$ denote that every $j \neq i$ chooses $a_{m}$.

The next lemma shows that for $p \leq 1$, only downward deviations can be profitable. Moreover, if profitable deviations exist, the one-step downward deviation is one of those. Consequently, the symmetric pure-strategy profile $\left(a_{0}, \ldots, a_{0}\right)$ is always a Nash equilibrium.

Lemma 1. Let $p \leq 1$. If $a_{m-1}$ is not a profitable deviation from $\left(a_{m}, \ldots, a_{m}\right)$ then there is no profitable deviation, i.e., $\left(a_{m}, \ldots, a_{m}\right)$ is a Nash equilibrium. In particular:

(i) For an arbitrary $m \in\{2, \ldots, M\}$, if $n \in\{1, \ldots, m-1\}$ then $u_{i}\left(a_{n}, \bar{a}_{m}^{-i}\right) \geq u_{i}\left(a_{n-1}, \bar{a}_{m}^{-i}\right)$.

(ii) For an arbitrary $m \in\{0, \ldots, M-1\}$, if $n \in\{m+1, \ldots, M\}$ then $u_{i}\left(\bar{a}_{m}\right)>u_{i}\left(a_{n}, \bar{a}_{m}^{-i}\right)$.

Hence, for every $m \in\{1, \ldots, M\}$, the symmetric pure-strategy profile $\left(a_{m}, \ldots, a_{m}\right)$ is a Nash equilibrium if and only if the one-step downward deviation to $a_{m-1}$ is not profitable. In turn, this is the case if and only if $p \geq p_{m}$. This completes the proof of Theorem 1 (i).

The next lemma is analogous to Lemma 1 for $p \geq 1$. In particular, it shows that only upward deviations can be profitable. Furthermore, if there are profitable deviations, the one-step upward deviation is one of those. As a consequence, the symmetric pure-strategy profile $\left(a_{M}, \ldots, a_{M}\right)$ is always a Nash equilibrium.

Lemma 2. Let $p \geq 1$. If $a_{m+1}$ is not a profitable deviation from $\left(a_{m}, \ldots, a_{m}\right)$ then there is no profitable deviation, and therefore $\left(a_{m}, \ldots, a_{m}\right)$ is a Nash equilibrium. In particular: 
(i) For an arbitrary $m \in\{1, \ldots, M\}$, if $n \in\{0, \ldots, m-1\}$ then $u_{i}\left(\bar{a}_{m}\right)>u_{i}\left(a_{n}, \bar{a}_{m}^{-i}\right)$.

(ii) For an arbitrary $m \in\{0, \ldots, M-2\}$, if $n \in\{m+1, \ldots, M-1\}$ then $u_{i}\left(a_{n}, \bar{a}_{m}^{-i}\right) \geq u_{i}\left(a_{n+1}, \bar{a}_{m}^{-i}\right)$.

Therefore, for every $m \in\{0, \ldots, M-1\}$, the symmetric pure-strategy profile $\left(a_{m}, \ldots, a_{m}\right)$ is a Nash equilibrium if and only if the one-step upward deviation to $a_{m+1}$ is not profitable. This is the case whenever $p \leq q_{m}$, thus proving Theorem 1 (ii).

\subsection{Theorem 2: rationalizable strategies}

We will use the following two lemmas to prove that, if the symmetric strategy profiles $\left(a_{m}, \ldots, a_{m}\right)$ and $\left(a_{n}, \ldots, a_{n}\right)$ are Nash equilibria (with $m \leq n$ ), the (symmetric) product set

$$
C_{1}^{m, n} \times \cdots \times C_{N}^{m, n}
$$

is a best response set, where $C_{i}^{m, n}:=\left\{a_{m}, \ldots, a_{n}\right\} .^{4}$ This directly implies that every $s_{i} \in C_{i}^{m, n}$ is rationalizable. Then, for each of the two cases, $p \leq 1$ and $p \geq 1$, we take the "lowest" and the "highest" Nash equilibrium and we show that every strategy outside the range of these extreme Nash equilibria is eliminated by iterated strict dominance.

For notation simplicity, for each $m \in\{0, \ldots, M\}$ we define two reduced games of interest by $B_{i}^{m}=\left\{a_{0}, \ldots, a_{m}\right\}$ and $A_{i}^{m}=\left\{a_{m}, \ldots, a_{M}\right\}$, viz., $B_{1}^{m} \times \cdots \times B_{N}^{m}$ is the game that we obtain after having eliminated all the strategies that are larger than $m$, whereas $A_{1}^{m} \times \cdots \times A_{N}^{m}$ is the game that we obtain after having eliminated all the strategies that are smaller than $m$.

Lemma 3. Let $p \leq 1$ and fix an arbitrary $m \in\{1, \ldots, M\}$. The following statements hold:

(i) $a_{m-1}$ is a best response to some $s_{-i} \in S_{-i}$ with $s_{j} \in\left\{a_{m-1}, a_{m}\right\}$ for all $j \neq i$.

(ii) If $\left(a_{m}, \ldots, a_{m}\right)$ is not a Nash equilibrium, then $a_{m}$ is strictly dominated in $B_{1}^{m} \times \cdots \times B_{N}^{m}$.

By Theorem 1 (i), the "lowest" Nash equilibrium is $\left(a_{0}, \ldots, a_{0}\right)$. Therefore, once we find the "highest" Nash equilibrium $\left(a_{\bar{m}}, \ldots, a_{\bar{m}}\right)$, every $a_{n} \in\left\{a_{0}, \ldots, a_{\bar{m}}\right\}$ is rationalizable. Indeed, by Lemma 3 (i), $a_{\bar{m}}$ is a best response to everybody else choosing $a_{\bar{m}}, a_{\bar{m}-1}$ is a best response to some $s_{-i}$ such that everybody else chooses either $a_{\bar{m}}$ or $a_{\bar{m}-1}$, and so on until we reach $a_{0}$ which is a best response to everybody else choosing $a_{0}$.

Now we focus on strategies larger than the "highest" Nash equilibrium (assuming $M>a_{\bar{m}}$ ) to show that all of them are iteratively eliminated. In particular, since $\left(a_{M}, \ldots, a_{M}\right)$ is not a Nash equilibrium, it is strictly dominated by a mixed strategy in $\Delta\left(\left\{a_{0}, \ldots, a_{M-1}\right\}\right)$. This mixed strategy puts sufficiently high probability on $a_{M-1}$ and uniformly distributes the remaining mass to all other strategies. In fact, in the proof of Lemma 3 (ii), we explicitly identify this mixed strategy. We continue inductively, until all strategies $a_{n} \in\left\{a_{\bar{m}+1}, \ldots, a_{M}\right\}$ have been iteratively eliminated, thus completing the proof of Theorem 2 (i).

Lemma 4. Let $p \geq 1$ and fix an arbitrary $m \in\{0, \ldots, M-1\}$. The following statements hold:

(i) $a_{m+1}$ is a best response to some $s_{-i} \in S_{-i}$ with $s_{j} \in\left\{a_{m}, a_{m+1}\right\}$ for all $j \neq i$.

(ii) If $\left(a_{m}, \ldots, a_{m}\right)$ is not a Nash equilibrium, then $a_{m}$ is strictly dominated in $A_{1}^{m} \times \cdots \times A_{N}^{m}$.

Similar to the previous case, the "highest" Nash equilibrium is $\left(a_{M}, \ldots, a_{M}\right)$, and after finding the "lowest" Nash equilibrium $\left(a_{m}, \ldots, a_{m}\right)$, we show that every $a_{n} \in\left\{a_{m}, \ldots, a_{M}\right\}$ is rationalizable. Subsequently, we show that strategies larger than the "highest" Nash equilibrium are iteratively eliminated, thus completing the proof of Theorem 2 (ii).

\section{Discussion}

\subsection{Independent rationalizability}

As mentioned, we employ the notion of correlated rationalizability, which in general coarsens Bernheim's (1984) and Pearce's (1984) notion of (independent) rationalizability. Nevertheless, as we show below, the two concepts yield the same predictions for guessing games.

For each player $i \in I$, let $S_{i}^{0}:=S_{i}$ and inductively define the strategies $S_{i}^{k} \subseteq S_{i}$ that survive $k$ rounds of elimination of Bernheim's (1984) and Pearce's (1984) procedure (a formal definition of $S_{i}^{k}$ is presented in the Appendix). Then, $S_{i}^{*}:=$ $\bigcap_{k=1}^{\infty} S_{i}^{k}$ consists of the independently rationalizable strategies.

\footnotetext{
${ }^{4}$ A product set $C_{1} \times \cdots \times C_{N} \subseteq S_{1} \times \cdots \times S_{N}$ is a best response set whenever every $s_{i} \in C_{i}$ is rational for some belief in $\Delta\left(C_{-i}\right)$. A formal definition is presented in the appendix.
} 
Proposition 1. For every $p \in \mathbb{R}_{+}$, a strategy is independently rationalizable if and only if it is (correlated) rationalizable, i.e., formally $R_{i}^{*}=S_{i}^{*}$.

Intuitively the proof follows directly from Lemma 3 (for $p \leq 1$ ) and Lemma 4 (for $p \geq 1$ ). In particular, we show that the best response set in (1) is an independent best response set, as each strategy in $C_{i}^{m, n}$ is a best response to a pure strategy profile in $C_{-i}^{m, n}$, i.e., it is rational to a product measure over $C_{-i}^{m, n}$.

\subsection{Rounds of elimination}

It is well-known that a strategy survives $k$ rounds of elimination of strictly dominated strategies if and only if it can be rationally played under $(k-1)$-fold belief in rationality. Since the game is often used to illustrate iterative reasoning, we want to see which strategies survive $k$ rounds of elimination. The following result characterizes each step of the elimination procedure. Recall that the set of rationalizable strategies is of the form of $R_{i}^{*}=\left\{a_{0}, \ldots, a_{\bar{m}}\right\}$ when $p \leq 1$ and of the form of $R_{i}^{*}=\left\{a_{\underline{m}}, \ldots, a_{M}\right\}$ when $p \geq 1$.

Proposition 2. Fix an arbitrary $p \in \mathbb{R}_{+}$. At each round of elimination of strictly dominated strategies, at most one strategy is deleted. In particular, the following statements hold:

(i) Fix $p \leq 1$. Then, $R_{i}^{k+1}=R_{i}^{k} \backslash\left\{a_{M-k}\right\}$ for all $k<M-\bar{m}$, and $R_{i}^{k+1}=R_{i}^{k}$ for all $k \geq M-\bar{m}$.

(ii) Fix $p \geq 1$. Then, $R_{i}^{k+1}=R_{i}^{k} \backslash\left\{a_{k}\right\}$ for all $k<\underline{m}$, and $R_{i}^{k+1}=R_{i}^{k}$ for all $k \geq \underline{m}$.

For $p \leq 1$ (resp., for $p \geq 1$ ), as long as there are still strategies that are not rationalizable, we can only eliminate the highest (resp., the lowest) remaining strategy, since the second highest (resp., the second lowest) is still rational against the strategies that remain, by Lemma 3 (i) (resp., by Lemma 4 (i)). Thus, the elimination process is very slow, i.e., depending on the parameters, a high belief in rationality might be needed to end up with the set of rationalizable strategies.

\subsection{The discrete limit and continuous strategy sets}

In this section, we illustrate what happens as the difference between the guesses converges to zero and we consider the case of continuous strategies. We focus on the case $p \leq 1$ and strategy sets of the form $S_{i}=\{0, \Delta, 2 \Delta, \ldots, M\}$, where $M$ is an integer-multiple of $\Delta$. In this case, the threshold such that the strategy profile $(s, \ldots, s)$ is a Nash equilibrium and therefore $s$ is rationalizable is $p_{s} \geq \frac{2 N s-N \Delta}{2 N s-2 \Delta}$.

Intuitively, in the main text, if there are many players, a downward deviation by a unit might not drag the target guess enough towards $s-\Delta$ to make this deviation profitable. As the step size goes to zero, however, this effect disappears: note that $\lim _{\Delta \rightarrow 0} p_{s}=1$, i.e., strategy $s$ is not rationalizable for any $p<1$.

Finally, suppose we start with a continuous strategy set $S_{i}=[0, M]$ where $M$ is a positive real number. In this case, it is straightforward that the only symmetric Nash equilibrium is $(0, \ldots, 0)$ for $p<1$. For rationalizability, the main difficulty is that Pearce's (1984) Lemma does not directly extend to infinite games. Hence, we cannot conclude that eliminating strictly dominated strategies is equivalent to eliminating strategies that are not rational for any belief. Let us focus on eliminating at each round strategies that are not rational. ${ }^{5}$ Then for an arbitrary $p<1$, only $M$ is eliminated at the first round. Moreover, at the second round, all strategies are rational. Indeed, for each $m<M$, there is some $n \in(m, M)$ such that $m$ is rational to everybody else choosing $n$. Hence, every $m \in[0, M)$ is rationalizable.

\section{Appendix A}

Let us first introduce some additional notation. Recall that $I=\{1, \ldots, N\}$ and $S_{i}:=\left\{a_{0}, a_{1}, \ldots, a_{M}\right\}$ with $N>2$ and $M \geq 2$. We define $S:=\chi_{i \in I} S_{i}$ with typical element $s=\left(s_{1}, \ldots, s_{N}\right)$, and $S_{-i}:=\chi_{j \neq i} S_{j}$ with typical element $s_{-i}=$ $\left(s_{1}, \ldots, s_{i-1}, s_{i+1}, \ldots, s_{N}\right)$. Moreover, let us denote by $\bar{a}_{m} \in S$ the symmetric strategy profile according to which $s_{i}=a_{m}$ for all $i \in I$, and by $\bar{a}_{m}^{-i} \in S_{-i}$ the strategy profile of the opponents where $s_{j}=a_{m}$ for every $j \neq i$. As usual, a reduced game is defined by means of a product set $C=C_{1} \times \cdots \times C_{N}$ where $C_{i} \subseteq S_{i}$ for each player $i \in I$, together with the original utility function restricted to the set $C$. As usual we define $C_{-i}:=X_{j \neq i} C_{j}$. For each $m \in\{0, \ldots, M\}$ we define two (symmetric) reduced games of interest, viz., we take $C_{i}=B_{i}^{m}$ and $C_{i}=A_{i}^{m}$, with $B_{i}^{m}=\left\{a_{0}, \ldots, a_{m}\right\}$ and $A_{i}^{m}=\left\{a_{m}, \ldots, a_{M}\right\}$ respectively.

Recall that all players are expected utility maximizers. Since there are only two outcomes - i.e., winning and losing the prize - we can normalize without loss of generality the respective vNM utility indexes to 0 and 1 . Formally, for an arbitrary $s \in S$ we define the target guess

$$
t(s)=\frac{p}{N} \sum_{j \in I} s_{j},
$$

\footnotetext{
${ }^{5}$ This procedure is common in the literature on games with continuous strategy spaces (e.g., see Arieli, 2010).
} 
and the distance from the target guess

$$
d_{i}(s):=\left|s_{i}-t(s)\right|,
$$

with $W(s):=\left\{i \in I: d_{i}(s) \leq d_{j}(s)\right.$ for all $\left.j \in I\right\}$ being the respective set of winners. Then, the vNM utility of player $i \in I$ from a pure strategy profile $s \in S$ is given by $u_{i}(s):=\frac{1}{|W(s)|}$ if $i \in W(s)$ and $u_{i}(s)=0$ otherwise.

A strategy profile $\left(s_{1}, \ldots, s_{N}\right)$ is a Nash equilibrium if $u_{i}\left(s_{i}, s_{-i}\right) \geq u_{i}\left(s_{i}^{\prime}, s_{-i}\right)$ for every $s_{i}^{\prime} \in S_{i}$ and every $i \in I$.

We define a mixed strategy $\sigma_{i} \in \Delta\left(S_{i}\right)$ as a randomization over $i$ 's own strategies, and define $i$ 's expected utility from $\sigma_{i}$ given some $s_{-i}$ by $u_{i}\left(\sigma_{i}, s_{-i}\right)=\sum_{s_{i} \in S_{i}} \sigma_{i}\left(s_{i}\right) \cdot u_{i}\left(s_{i}, s_{-i}\right)$. We define a belief $\mu_{i} \in \Delta\left(S_{-i}\right)$ of player $i$ as a probability measure over the opponents' strategy profiles, and we define $i$ 's expected utility from $s_{i}$ given $\mu_{i}$, by $u_{i}\left(s_{i}, \mu_{i}\right)=\sum_{s_{-i} \in S_{-i}} \mu_{i}\left(s_{-i}\right)$. $u_{i}\left(s_{i}, s_{-i}\right)$. A belief $\mu_{i}$ is called independent if there exists some mixed strategy profile $\left(\sigma_{1}, \ldots, \sigma_{i-1}, \sigma_{i+1}, \ldots, \sigma_{N}\right)$ of $i$ 's opponents such that $\mu_{i}=\bigotimes_{j \neq i} \sigma_{j}$, i.e., if $\mu_{i}$ is a product measure assigning probability $\mu_{i}\left(s_{-i}\right)=\prod_{j \neq i} \sigma_{j}\left(s_{j}\right)$ to each $s_{-i}=\left(s_{1}, \ldots, s_{i-1}, s_{i+1}, \ldots, s_{N}\right) \in S_{-i}$. The belief is called correlated if it is not independent. A strategy $s_{i} \in S_{i}$ is called rational in the game $C_{1} \times \cdots \times C_{N}$ if there exists some - not necessarily independent - belief $\mu_{i} \in \Delta\left(C_{-i}\right)$ such that $u_{i}\left(s_{i}, \mu_{i}\right) \geq u_{i}\left(s_{i}^{\prime}, \mu_{i}\right)$ for all $s_{i}^{\prime} \in C_{i}$. It follows from Pearce's (1984) Lemma that $s_{i}$ is rational in $C_{1} \times \cdots \times C_{N}$ if and only if it is not strictly dominated by any mixed strategy in $C_{1} \times \cdots \times C_{N}$, i.e., if and only if there is no $\sigma_{i} \in \Delta\left(C_{i}\right)$ such that $u_{i}\left(\sigma_{i}, s_{-i}\right)>u_{i}\left(s_{i}, s_{-i}\right)$ for all $s_{-i} \in C_{-i}$.

For each $i \in I$, we let $R_{i}^{0}:=S_{i}$, and for each $k>0$ we inductively define the strategies $R_{i}^{k}:=\left\{s_{i} \in R_{i}^{k-1}: s_{i}\right.$ is rational in $\left.R_{1}^{k-1} \times \cdots \times R_{N}^{k-1}\right\}$ surviving $k$ rounds of elimination of strictly dominated strategies. Then, $R_{i}^{*}:=\bigcap_{k=0}^{\infty} R_{i}^{k}$ consists of the (correlated) rationalizable strategies. For each $i \in I$, we let $S_{i}^{0}:=S_{i}$, and for each $k>0$ we inductively define the set $S_{i}^{k}:=\left\{s_{i} \in S_{i}^{k-1}: s_{i}\right.$ is rational for some independent belief in $\left.S_{1}^{k-1} \times \cdots \times S_{N}^{k-1}\right\}$. Then, $S_{i}^{*}:=\bigcap_{k=0}^{\infty} S_{i}^{k}$ consists of the (independent) rationalizable strategies à la Bernheim (1984) and Pearce (1984).

A reduced game $C_{1} \times \cdots \times C_{N}$ is a best response set (resp., independent best response set) whenever every $s_{i} \in C_{i}$ is rational given some belief (resp., given some independent belief) $\mu_{i} \in \Delta\left(C_{-i}\right)$. Then, it is well known that if $C_{1} \times \cdots \times C_{N}$ is a best response set (resp., an independent best response set) then $C_{i} \subseteq R_{i}^{*}$ (resp., $C_{i} \subseteq S_{i}^{*}$ ) for every $i \in I$.

\section{A.1. Intermediate results}

Lemma A.1. For every $s_{-i} \in S_{-i}$ there exists some $s_{i} \in S_{i}$ such that $u_{i}\left(s_{i}, s_{-i}\right) \geq 1 / N$.

Proof. Since at most $N$ players tie, we have $u_{i}\left(s_{i}, s_{-i}\right)>0$ implies $u_{i}\left(s_{i}, s_{-i}\right) \geq 1 / N$. Thus, it suffices to show $u_{i}\left(s_{i}, s_{-i}\right)>0$. Fix an arbitrary $s_{-i} \in S_{-i}$ and define the function $\delta_{i}\left(s_{i}\right):=s_{i}-t\left(s_{i}, s_{-i}\right)$, which is linear and therefore continuous in $\mathbb{R}_{+}$. Consider the following three cases:

- $\delta_{i}\left(a_{0}\right) \geq 0$ : Then, $s_{j} \geq a_{0} \geq t(s)$ for all $j \neq i$, and therefore $u_{i}\left(a_{0}, s_{-i}\right)>0$.

- $\delta_{i}\left(a_{M}\right) \leq 0$ : Then, $s_{j} \leq a_{M} \leq t(s)$ for all $j \neq i$, and therefore $u_{i}\left(a_{M}, s_{-i}\right)>0$.

- $\delta_{i}\left(a_{0}\right)<0$ and $\delta_{i}\left(a_{M}\right)>0$ : By the intermediate value theorem, there exists some $a \in\left(a_{0}, a_{M}\right)$ such that $\delta_{i}(a)=0$. Obviously if $a \in S_{i}$ then $u_{i}\left(a, s_{-i}\right)>0$ and we are done. Hence, let us take $a \notin S_{i}$. We define $a_{m}:=\max \left\{s_{i} \in S_{i}: s_{i}<a\right\}$ and $a_{m+1}:=\min \left\{s_{i} \in S_{i}: s_{i}>a\right\}$. Consider the following three subcases:

○ $N=p$ : Then, $\delta_{i}$ becomes constant in $s_{i}$. In particular, $\delta_{i}\left(a_{0}\right)=\delta_{i}\left(a_{M}\right)$. Hence, we are back to one of the previous two cases.

$\circ N<p$ : Then, $\delta_{i}$ becomes strictly decreasing in $s_{i}$ and therefore $s_{i}<t\left(s_{i}, s_{-i}\right)$ for every $s_{i} \in\left\{a_{m+1}, \ldots, a_{M}\right\}$. Hence, $t\left(a_{M}, s_{-i}\right)>a_{M}$ which contradicts $\delta_{i}\left(a_{M}\right)>0$.

○ $N>p$ : Then, $\delta_{i}$ becomes strictly increasing in $s_{i}$ and therefore $a_{m}<t\left(a_{m}, s_{-i}\right)$ and $a_{m+1}>t\left(a_{m+1}, s_{-i}\right)$. Moreover, since $t$ is strictly increasing, $a_{m}<t\left(a_{m}, s_{-i}\right)<t\left(a_{m+1}, s_{-i}\right)<a_{m+1}$. Hence, at least one of the two guesses wins with positive probability, i.e., either $u_{i}\left(a_{m}, s_{-i}\right)>0$ (viz., if $\left.t\left(a_{m}, s_{-i}\right)<t\left(a_{m+1}, s_{-i}\right)<\frac{1}{2}\left(a_{m+1}-a_{m}\right)\right)$ or $u_{i}\left(a_{m+1}, s_{-i}\right)>0$ (viz., if $\left.\frac{1}{2}\left(a_{m+1}-a_{m}\right)<t\left(a_{m}, s_{-i}\right)<t\left(a_{m+1}, s_{-i}\right)\right)$ or both $u_{i}\left(a_{m}, s_{-i}\right)>0$ and $u_{i}\left(a_{m+1}, s_{-i}\right)>0$ (viz., if $t\left(a_{m}, s_{-i}\right)<$ $\left.\frac{1}{2}\left(a_{m+1}-a_{m}\right)<t\left(a_{m+1}, s_{-i}\right)\right)$.

Combining the previous cases completes the proof of the lemma.

Lemma A.2. Fix an arbitrary $p \leq 1$.

(i) Fix an arbitrary $m \in\{2, \ldots, M\}$ and an arbitrary $s_{-i} \in S_{-i}$ such that $s_{j} \geq a_{m}$ for every $j \neq i$. Then $u_{i}\left(a_{n}, s_{-i}\right) \geq u_{i}\left(a_{n-1}, s_{-i}\right)$ for all $n \in\{1, \ldots, m-1\}$.

(ii) Fix an arbitrary $m \in\{0, \ldots, M-1\}$ and an arbitrary $s_{-i} \in S_{-i}$ such that $s_{j} \leq a_{m}$ for all $j \neq i$. Then $u_{i}\left(a_{n}, s_{-i}\right)=0$ for all $n \in\{m+1, \ldots, M\}$.

Proof. (i) Fix arbitrary $m \in\{2, \ldots, M\}$ and $n \in\{1, \ldots, m-1\}$. Without loss of generality, take some $s_{-i}$ with $m=$ $\min \left\{s_{1}, \ldots, s_{i-1}, s_{i+1}, \ldots, s_{N}\right\}$. Then it is straightforward to verify that $u_{i}\left(a_{n}, s_{-i}\right) \geq \frac{1}{N}$ if and only if $d_{i}\left(a_{n}, s_{-i}\right) \leq d_{j}\left(a_{n}, s_{-i}\right)$ 
for an arbitrary $j \neq i$ such that $s_{j}=a_{m}$. Moreover, note that whenever the second inequality is strict, we obtain $u_{i}\left(a_{n}, s_{-i}\right)=1$. Observe that $d_{i}\left(a_{n}, s_{-i}\right) \leq d_{j}\left(a_{n}, s_{-i}\right)$ is equivalent to

$$
p \leq \frac{N}{2} \cdot \frac{a_{n}+a_{m}}{a_{n}+\sum_{j \neq i} s_{j}},
$$

which holds with equality if and only if $d_{i}\left(a_{n}, s_{-i}\right)=d_{j}\left(a_{n}, s_{-i}\right)$. Notice that (by $N>2$ and $\left.\sum_{j \neq i} s_{j} \geq(N-1) a_{m}>a_{m}\right)$, the right-hand side of (A.1) is strictly increasing in $n$. Hence, if (A.1) holds for $n-1$ then it also holds (with strict inequality) for $n$. In other words, if $u_{i}\left(a_{n-1}, s_{-i}\right)=0$ then it is trivially the case that $u_{i}\left(a_{n-1}, s_{-i}\right) \leq u_{i}\left(a_{n}, s_{-i}\right)$, whereas if $u_{i}\left(a_{n-1}\right.$, $\left.s_{-i}\right) \geq \frac{1}{N}$ it will necessarily be the case that $u_{i}\left(a_{n}, s_{-i}\right)=1$, thus completing this part of the proof.

(ii) Fix arbitrary $m \in\{0, \ldots, M-1\}$ and $n \in\{m+1, \ldots, M\}$, and without loss of generality let $m=\max \left\{s_{1}, \ldots, s_{i-1}, s_{i+1}\right.$, $\left.\ldots, s_{N}\right\}$. Then the target guess under the strategy profile $\left(a_{n}, s_{-i}\right)$ is

$$
\begin{aligned}
t\left(a_{n}, s_{-i}\right) & =\frac{p}{N}\left(a_{n}+\sum_{j \neq i} s_{j}\right) \\
& \leq \frac{1}{N}\left(a_{n}+(N-1) a_{m}\right) \\
& <\frac{1}{2}\left(a_{n}+a_{m}\right),
\end{aligned}
$$

with the second strict inequality holding due to $N>2$ and $a_{n}>a_{m}$. Hence, $d_{i}\left(a_{n}, s_{-i}\right)>d_{j}\left(a_{n}, s_{-i}\right)$ where $j \neq i$ is an opponent of $i$ such that $s_{j}=a_{m}$. Hence, $u_{i}\left(a_{n}, s_{-i}\right)=0$.

Lemma A.3. Fix an arbitrary $p \geq 1$.

(i) Fix an arbitrary $m \in\{0, \ldots, M-2\}$ and an arbitrary $s_{-i} \in S_{-i}$ such that $s_{j} \leq a_{m}$ for every $j \neq i$. Then $u_{i}\left(a_{n}, s_{-i}\right) \geq u_{i}\left(a_{n+1}, s_{-i}\right)$ for all $n \in\{m+1, \ldots, M-1\}$.

(ii) Fix an arbitrary $m \in\{1, \ldots, M\}$ and an arbitrary $s_{-i} \in S_{-i}$ such that $s_{j} \geq a_{m}$ for all $j \neq i$ and $u_{i}\left(a_{m}, s_{-i}\right)>0$. Then $u_{i}\left(a_{m}, s_{-i}\right)>$ $u_{i}\left(a_{n}, s_{-i}\right)$ for all $n \in\{1, \ldots, m-1\}$.

Proof. (i) The proof is similar to one of Lemma A.2 (i). Indeed fix arbitrary $m \in\{0, \ldots, M-2\}$ and $n \in\{m+1, \ldots, M-1\}$. Without loss of generality take some $s_{-i} \in S_{-i}$ such that $m=\max \left\{s_{1}, \ldots, s_{i-1}, s_{i+1}, \ldots, s_{N}\right\}$. Then, we obtain $u_{i}\left(a_{n}, s_{-i}\right) \geq \frac{1}{N}$ if and only if

$$
p \geq \frac{N}{2} \cdot \frac{a_{n}+a_{m}}{a_{n}+\sum_{j \neq i} s_{j}} .
$$

In particular, $\frac{1}{N} \leq u_{i}\left(a_{n}, s_{-i}\right)<1$ if and only if (A.2) holds with equality, whereas $u_{i}\left(a_{n}, s_{-i}\right)=1$ if and only if (A.2) holds with strict inequality. Whenever (A.2) does not hold, we obtain $u_{i}\left(a_{n}, s_{-i}\right)=0$. The right-hand side of (A.2) is strictly increasing in $n$. Hence, if $u_{i}\left(a_{n+1}, s_{-i}\right)=0$ then $u_{i}\left(a_{n+1}, s_{-i}\right) \leq u_{i}\left(a_{n}, s_{-i}\right)$ holds trivially, whereas if $u_{i}\left(a_{n+1}, s_{-i}\right) \geq \frac{1}{N}$ then $u_{i}\left(a_{n}, s_{-i}\right)=1$, thus completing this part of the proof.

(ii) The proof is identical to the one of Lemma A.2 (ii). For every $m \in\{1, \ldots, M\}$ and every $n \in\{0, \ldots, m-1\}$, without loss of generality take some $s_{-i} \in S_{-i}$ with $m=\min \left\{s_{1}, \ldots, s_{i-1}, s_{i+1}, \ldots, s_{N}\right\}$. Then, we obtain $t\left(a_{n}, s_{-i}\right)>\frac{1}{2}\left(a_{n}+a_{m}\right)$ using the same argument as above. Hence, $d_{i}\left(a_{n}, s_{-i}\right)>d_{j}\left(a_{n}, s_{-i}\right)$ where $j \neq i$ is an opponent of $i$ such that $s_{j}=a_{m}$, and therefore $u_{i}\left(a_{n}, s_{-i}\right)=0$.

\section{A.2. Proofs of Section 4.1}

Proof of Lemma 1. The result is a direct corollary of Lemma A.2 for $s_{j}=a_{m}$ for all $j \neq i$.

Proof of Lemma 2. The result is a direct corollary of Lemma A.3 for $s_{j}=a_{m}$ for all $j \neq i$.

Proof of Theorem 1. Let $\left(s_{1}, \ldots, s_{N}\right)$ be a Nash equilibrium. By Lemma A.1, there exists some $s_{i}^{*} \in S_{i}$ such that $u_{i}\left(s_{i}^{*}, s_{-i}\right) \geq$ $1 / N$, and therefore $u_{i}\left(s_{i}, s_{-i}\right) \geq u_{i}\left(s_{i}^{*}, s_{-i}\right) \geq 1 / N$. Moreover, by the rules of the game, $\sum_{i \in I} u_{i}\left(s_{1}, \ldots, s_{N}\right)=1$, thus implying $u_{i}\left(s_{1}, \ldots, s_{N}\right)=1 / N$ for every $i \in I$. Hence $\left|s_{i}-t\left(s_{1}, \ldots, s_{N}\right)\right|=c \in \mathbb{R}_{+}$for every $i \in I$. Thus, there are at most two guesses $a_{m}, a_{n} \in S_{i}$ such that $s_{i} \in\left\{a_{m}, a_{n}\right\}$ for every $i \in I$. Without loss of generality let $a_{m}<a_{n}$. Suppose - contrary to what we want to prove - that there exists a strict nonempty subset $J \subsetneq I$ of the players choosing $a_{m}$ and the rest choosing $a_{n}$ i.e., assume that $J=\left\{i \in I: s_{i}=a_{m}\right\}$ and $I \backslash J=\left\{i \in I: s_{i}=a_{n}\right\}$, with $0<|J|<N$. Hence, the target guess $t\left(s_{1}, \ldots, s_{N}\right)=$ $\frac{p}{N}\left(|J| a_{m}+(N-|J|) a_{n}\right)$ is equal to $\left(a_{m}+a_{n}\right) / 2$. Since $N>2$, we obtain $|J| \geq 2$ or $N-|J| \geq 2$. If $|J| \geq 2$, let $i \in J$ switch from $a_{m}$ to $a_{n}$, in which case the target guess satisfies 


$$
\begin{aligned}
t\left(s_{1}, \ldots, s_{i-1}, a_{n}, s_{i+1}, \ldots, s_{N}\right) & =\frac{p}{N}\left((|J|-1) a_{m}+(N-|J|+1) a_{n}\right) \\
& >\frac{p}{N}\left(|J| a_{m}+(N-|J|) a_{n}\right) \\
& =\frac{1}{2}\left(a_{m}+a_{n}\right) .
\end{aligned}
$$

Thus, the $|J|-1$ players in $J \backslash\{i\}$ do not win and $u_{i}\left(s_{1}, \ldots, s_{i-1}, a_{n}, s_{i+1}, \ldots, s_{N}\right)=\frac{1}{N-|J|+1}>\frac{1}{N}=u_{i}\left(s_{1}, \ldots, s_{N}\right)$, which contradicts $\left(s_{1}, \ldots, s_{N}\right)$ being a Nash equilibrium. Similarly, if $N-|J| \geq 2$, any deviation of a player $j \in I \backslash J$ from $a_{n}$ to $a_{m}$ is profitable, a contradiction to $\left(s_{1}, \ldots, s_{N}\right)$ being a Nash equilibrium. Therefore, any Nash equilibrium is symmetric, i.e., $s_{i}=a_{m}$ for all $i \in I$.

(i) It follows from Lemma 1 (ii) that $\left(a_{0}, \ldots, a_{0}\right)$ is always a Nash equilibrium. Thus, by our convention $p_{0}=0$, it follows directly that $\left(a_{0}, \ldots, a_{0}\right)$ is a Nash equilibrium if and only if $p \geq p_{0}$. Fix an arbitrary $m \in\{1, \ldots, M\}$. By Lemma 1 , the strategy profile $\left(a_{m}, \ldots, a_{m}\right)$ is a Nash equilibrium if and only if $d_{i}\left(a_{m-1}, \bar{a}_{m}^{-i}\right) \geq d_{j}\left(a_{m-1}, \bar{a}_{m}^{-i}\right)$. The latter is equivalent to $\frac{p}{N}\left(a_{m-1}+(N-1) a_{m}\right) \geq \frac{1}{2}\left(a_{m-1}+a_{m}\right)$, which is in turn equivalent to $p \geq p_{m}$.

(ii) It follows from Lemma 2 (ii) that $\left(a_{M}, \ldots, a_{M}\right)$ is always a Nash equilibrium. Thus, by our convention $p_{M}=\infty$, the profile $\left(a_{M}, \ldots, a_{M}\right)$ is a Nash equilibrium if and only if $p \leq p_{M}$. Now fix an arbitrary $m \in\{0, \ldots, M-1\}$. Then, by Lemma 2 , the strategy profile $\left(a_{m}, \ldots, a_{m}\right)$ is a Nash equilibrium if and only if $d_{i}\left(a_{m+1}, \bar{a}_{m}^{-i}\right) \geq d_{j}\left(a_{m+1}, \bar{a}_{m}^{-i}\right)$, which is equivalent to $\frac{p}{N}\left(a_{m+1}+(N-1) a_{m}\right) \leq \frac{1}{2}\left(a_{m+1}+a_{m}\right)$, which is in turn equivalent to $p \leq q_{m}$, thus completing the proof.

\section{A.3. Proofs of Section 4.2}

Lemma A.4. For an arbitrary $m \in\{1, \ldots, M\}$, let $p<p_{m}$ and consider an arbitrary $s_{-i} \in B_{-i}^{m}$. If $u_{i}\left(a_{m}, s_{-i}\right)>0$ then $u_{i}\left(a_{m-1}, s_{-i}\right)>$ $u_{i}\left(a_{m}, s_{-i}\right)$.

Proof. Fix an arbitrary $s_{-i} \in B_{-i}^{m}$ such that $u_{i}\left(a_{m}, s_{-i}\right)>0$. First, it follows by Theorem 1 (i) that $s_{-i} \neq \bar{a}_{m}^{-i}$, i.e., there exists some $k \neq i$ such that $s_{k}=a_{n}<a_{m}$. Without loss of generality let $a_{n} \geq s_{j}$ for every $j \neq i$ such that $s_{j} \neq a_{m}$, i.e., among the opponents of $i$ who do not choose $a_{m}$, player $k$ chooses the highest number. Then it follows from $u_{i}\left(a_{m}, s_{-i}\right)>0$, that $t\left(a_{m}, s_{-i}\right) \geq \frac{1}{2}\left(a_{m}+a_{n}\right)$. Let us first prove that $a_{n}<a_{m-1}$. Suppose not, i.e., assume that $a_{n}=a_{m-1}$, in which case

$$
\begin{aligned}
t\left(a_{m}, s_{-i}\right) & =\frac{p}{N}\left(a_{m}+\sum_{j \neq i} s_{j}\right) \\
& \leq \frac{p}{N}\left((N-1) a_{m}+a_{m-1}\right) \\
& =t\left(a_{m-1}, \bar{a}_{m}^{-i}\right) \\
& <\frac{1}{2}\left(a_{m}+a_{m-1}\right),
\end{aligned}
$$

where the last inequality follows from the fact that $\left(a_{m}, \ldots, a_{m}\right)$ is not a Nash equilibrium (see Theorem 1$)$ together with the fact that the only candidate for a profitable deviation is $a_{m-1}$ (see Lemma 1). But then, there exists a player choosing $a_{m-1}$, thus contradicting $u_{i}\left(a_{m}, s_{-i}\right)>0$. Hence, it must necessarily be the case that $a_{n}<a_{m-1}$, i.e., no opponent chooses $a_{m-1}$. Now consider the strategy profile $\left(a_{m-1}, s_{-i}\right)$ and obtain

$$
\begin{aligned}
t\left(a_{m-1}, s_{-i}\right) & =t\left(a_{m}, s_{-i}\right)-\frac{p}{N}\left(a_{m}-a_{m-1}\right) \\
& >\frac{1}{2}\left(a_{m}+a_{n}\right)-\frac{1}{2}\left(a_{m}-a_{m-1}\right) \\
& =\frac{1}{2}\left(a_{m-1}+a_{n}\right),
\end{aligned}
$$

implying that $d_{i}\left(a_{m-1}, s_{-i}\right)<d_{j}\left(a_{m-1}, s_{-i}\right)$ for every $j \neq i$ with $s_{j}<a_{m-1}$. Finally, notice that

$$
\begin{aligned}
t\left(a_{m-1}, s_{-i}\right) & <t\left(a_{m-1}, \bar{a}_{m}^{-i}\right) \\
& <\frac{1}{2}\left(a_{m-1}+a_{m}\right),
\end{aligned}
$$

with the second inequality following from $\bar{a}_{m}$ not being a Nash equilibrium. Hence, $d_{i}\left(a_{m-1}, s_{-i}\right)<d_{j}\left(a_{m-1}, s_{-i}\right)$ for every $j \neq i$ with $s_{j}>a_{m-1}$. This implies that $u_{i}\left(a_{m-1}, s_{-i}\right)=1$. Thus, it suffices to prove that $u_{i}\left(a_{m}, s_{-i}\right)<1$. Suppose otherwise, i.e., let $s_{j} \leq a_{n}$ for every $j \neq i$. Then, from Lemma A.2, we would obtain $u_{i}\left(a_{m}, s_{-i}\right)=0$, thus completing the proof. 
Lemma A.5. For an arbitrary $m \in\{0, \ldots, M-1\}$, let $p>q_{m}$ and consider an arbitrary $s_{-i} \in A_{-i}^{m}$. If $u_{i}\left(a_{m}, s_{-i}\right)>0$ then $u_{i}\left(a_{m+1}, s_{-i}\right)>u_{i}\left(a_{m}, s_{-i}\right)$.

Proof. The proof is very similar to the one of A.4 above. Fix an arbitrary $s_{-i} \in A_{-i}^{m}$ such that $u_{i}\left(a_{m}, s_{-i}\right)>0$. First, it follows by Theorem 1 (ii) that $s_{-i} \neq \bar{a}_{m}^{-i}$. Without loss of generality let $a_{n} \leq s_{j}$ for every $j \neq i$ such that $s_{j} \neq a_{m}$. Then it follows from $u_{i}\left(a_{m}, s_{-i}\right)>0$, that $t\left(a_{m}, s_{-i}\right) \leq \frac{1}{2}\left(a_{m}+a_{n}\right)$. Using the same arguments as above, we first show that $a_{n}>a_{m+1}$. Now consider the strategy profile $\left(a_{m+1}, s_{-i}\right)$ to obtain

$$
\begin{aligned}
t\left(a_{m+1}, s_{-i}\right) & =t\left(a_{m}, s_{-i}\right)-\frac{p}{N}\left(a_{m+1}-a_{m}\right) \\
& <\frac{1}{2}\left(a_{m+1}+a_{n}\right),
\end{aligned}
$$

implying that $d_{i}\left(a_{m+1}, s_{-i}\right)<d_{j}\left(a_{m+1}, s_{-i}\right)$ for every $j \neq i$ with $s_{j}>a_{m+1}$. Finally, notice that

$$
\begin{aligned}
t\left(a_{m+1}, s_{-i}\right) & <t\left(a_{m+1}, \bar{a}_{m}^{-i}\right) \\
& <\frac{1}{2}\left(a_{m-1}+a_{m}\right),
\end{aligned}
$$

with the second inequality following from $\bar{a}_{m}$ not being a Nash equilibrium. Hence, $d_{i}\left(a_{m+1}, s_{-i}\right)<d_{j}\left(a_{m+1}, s_{-i}\right)$ for every $j \neq i$ with $s_{j}<a_{m+1}$. This implies that $u_{i}\left(a_{m+1}, s_{-i}\right)=1$. Hence, it suffices to prove that $u_{i}\left(a_{m}, s_{-i}\right)<1$. Suppose otherwise, i.e., let $s_{j} \geq a_{n}$ for every $j \neq i$. Then, from Lemma A.3, we would obtain $u_{i}\left(a_{m}, s_{-i}\right)=0$, which completes the proof.

Proof of Lemma 3. (i) Fix some $m \in\{1, \ldots, M\}$ and define a strategy profile

$$
\underline{s}_{m}^{\kappa}=(\underbrace{a_{m}, \ldots, a_{m}}_{N-\kappa-1 \text { times }}, \underbrace{a_{m-1}, \ldots, a_{m-1}}_{\kappa \text { times }}) \in S_{-i},
$$

where $\kappa \in\{0, \ldots, N-1\}$ of $i$ 's opponents choose $a_{m-1}$ and the remaining ones choose $a_{m}$. Then, it suffices to prove that $a_{m-1}$ is a best response to some $s_{-i} \in\left\{\underline{s}_{m}^{0}, \underline{s}_{m}^{1}, \ldots, \underline{s}_{m}^{N-1}\right\}$. If $\left(a_{m}, \ldots, a_{m}\right)$ is not a Nash equilibrium, $a_{m-1}$ is a best response to $\underline{s}_{m}^{0}=\bar{a}_{m}^{-i}$ (by Lemma 1). If $\left(a_{m-1}, \ldots, a_{m-1}\right)$ is a Nash equilibrium, $a_{m-1}$ is trivially a best response to $\underline{s}_{m}^{N-1}=\bar{a}_{m-1}^{-i}$. So, we focus on the case where $\left(a_{m}, \ldots, a_{m}\right)$ is a Nash equilibrium while $\left(a_{m-1}, \ldots, a_{m-1}\right)$ is not. In particular, this implies $p<1$. Consider the strictly decreasing sequence

$$
\left(t\left(a_{m}, \underline{s}_{m}^{0}\right), t\left(a_{m-1}, \underline{s}_{m}^{0}\right), t\left(a_{m-1}, \underline{s}_{m}^{1}\right), \ldots, t\left(a_{m-1}, \underline{s}_{m}^{N-1}\right)\right)
$$

of the target guesses. Note that the difference

$$
t\left(a_{m}, \underline{s}_{m}^{0}\right)-t\left(a_{m-1}, \underline{s}_{m}^{0}\right)=t\left(a_{m-1}, \underline{s}_{m}^{\kappa}\right)-t\left(a_{m-1}, \underline{s}_{m}^{\kappa+1}\right)=\frac{p}{N}\left(a_{m}-a_{m-1}\right)
$$

does not depend on $\kappa$, i.e., the sequence decreases at a constant rate. Moreover, since $\left(a_{m}, \ldots, a_{m}\right)$ is a Nash equilibrium,

$$
a_{m} \geq t\left(a_{m}, \underline{s}_{m}^{0}\right)>t\left(a_{m-1}, \underline{s}_{m}^{0}\right) \geq \frac{1}{2}\left(a_{m}+a_{m-1}\right)>a_{m-1} \geq t\left(a_{m-1}, \underline{s}_{m}^{N-1}\right) .
$$

Define $\kappa^{*}:=\min \left\{\kappa \in\{1, \ldots, N-1\}: \frac{1}{2}\left(a_{m}+a_{m-1}\right)>t\left(a_{m-1}, \underline{s}_{m}^{\kappa}\right) \geq a_{m-1}\right\}$. Intuitively, this is the smallest number of opponents that must choose $a_{m-1}$ such that choosing $a_{m-1}$ yields a strictly higher utility than $a_{m}$. Note that by (A.3) and (A.4), we obtain $\frac{p}{N} \leq \frac{1}{2}$, and therefore such a $\kappa$ exists (the fact that $\kappa \geq 1$ follows from $\bar{a}_{m}$ being a Nash equilibrium). Consider the following cases:

- Let $\kappa^{*} \leq \frac{N-1}{2}$ or equivalently $\kappa^{*} \leq N-1-\kappa^{*}$, i.e., there are (weakly) fewer opponents choosing $a_{m-1}$ than those choosing $a_{m}$ at $\underline{s}_{m}^{\kappa^{*}}$. Let us first prove that

$$
u_{i}\left(a_{m-1}, \underline{s}_{m}^{\kappa^{*}}\right) \geq u_{i}\left(a_{n}, \underline{s}_{m}^{\kappa^{*}}\right)
$$

for all $n \in\{m+1, \ldots, M\}$. Observe that

$$
u_{i}\left(a_{m-1}, \underline{s}_{m}^{\kappa^{*}}\right)=\frac{1}{\kappa^{*}+1} \geq \frac{1}{N-\kappa^{*}}=u_{i}\left(a_{m}, \underline{s}_{m}^{\kappa^{*}}\right) .
$$

Moreover, by Lemma A.2 (ii), it follows that (A.5) holds for every $n \in\{m+1, \ldots, M\}$. Now, let us prove it for every $n \in\{0, \ldots, m-2\}$. By construction, $t\left(a_{m-1}, \underline{s}_{m}^{\kappa^{*}}\right) \geq a_{m-1}$. Hence, by $p \leq 1$ and $N>2$, 


$$
\begin{aligned}
t\left(a_{m-2}, \underline{s}_{m}^{\kappa^{*}}\right) & =t\left(a_{m-1}, \underline{s}_{m}^{\kappa^{*}}\right)-\frac{p}{N}\left(a_{m-1}-a_{m-2}\right) \\
& \geq a_{m-1}-\frac{p}{N}\left(a_{m-1}-a_{m-2}\right) \\
& >a_{m-1}-\frac{1}{2}\left(a_{m-1}-a_{m-2}\right) \\
& =\frac{1}{2}\left(a_{m-1}+a_{m-2}\right)
\end{aligned}
$$

implying that $d_{i}\left(a_{m-2}, \underline{s}_{m}^{\kappa^{*}}\right)>d_{j}\left(a_{m-2}, \underline{s}_{m}^{\kappa^{*}}\right)$, with $j \neq i$ being an opponent of $i$ who chooses $a_{m-1}$ (recall that such $j$ exists, since $\left.\kappa^{*} \geq 1\right)$. Hence, $u_{i}\left(a_{m-1}, \underline{s}_{m}^{\kappa^{*}}\right)>u_{i}\left(a_{m-2}, \underline{s}_{m}^{\kappa^{*}}\right)$, and thus (A.5) follows for all $n \in\{0, \ldots, m-2\}$ by Lemma A.2 (i). This implies that $a_{m-1}$ is a best response to $\underline{\kappa}_{m}^{\kappa^{*}} \in\left\{s_{-i} \in S_{-i}: s_{j} \in\left\{a_{m-1}, a_{m}\right\}\right.$ for all $\left.j \neq i\right\}$.

- Let $\kappa^{*}>\frac{N-1}{2}$, i.e., more than half of the opponents choose $a_{m-1}$. Since $\kappa^{*} \in \mathbb{N}$, we have $\kappa^{*} \geq N-\kappa^{*}$. Since additionally $p<1$, we obtain $t\left(a_{m-1}, \underline{s}_{m}^{\kappa^{*}-1}\right)=p \frac{\kappa^{*} a_{m-1}+\left(N-\kappa^{*}\right) a_{m}}{N}<\frac{a_{m}+a_{m-1}}{2}$, a contradiction to the definition of $\kappa^{*}$.

Combining the previous cases completes the proof.

(ii) Consider the mixed strategy $\sigma_{i}^{m} \in \Delta\left(B_{i}^{m}\right)$, attaching probability $\lambda \in\left(\frac{N}{1+N}, 1\right)$ to $a_{m-1}$ and equal probability $\frac{1-\lambda}{m-1}$ to each $a_{n} \in\left\{a_{0}, \ldots, a_{m-2}\right\}$. Then, consider the following two cases:

- Let $s_{-i} \in B_{-i}^{m}$ be such that $u_{i}\left(a_{m}, s_{-i}\right)=0$. Then, by Lemma A.1 applied in the reduced game $B_{1}^{m} \times \cdots \times B_{N}^{m}$, we obtain

$$
u_{i}\left(\sigma_{i}^{m}, s_{-i}\right)=\lambda u_{i}\left(a_{m-1}, s_{-i}\right)+\frac{1-\lambda}{m-1} \sum_{n=0}^{m-2} u_{i}\left(a_{n}, s_{-i}\right)>0=u_{i}\left(a_{m}, s_{-i}\right) .
$$

Indeed, for every $s_{-i} \in B_{-i}^{m}$ there exists some $n \in\{0, \ldots, m\}$ with $u_{i}\left(a_{n}, s_{-i}\right)>0$, and since $u_{i}\left(a_{m}, s_{-i}\right)=0$ it must be the case that $u_{i}\left(a_{n}, s_{-i}\right)>0$ for some $n \in\{0, \ldots, m-1\}$.

- Let $s_{-i} \in B_{-i}^{m}$ be such that $u_{i}\left(a_{m}, s_{-i}\right)>0$. Since $\left(a_{m}, \ldots, a_{m}\right)$ is not a Nash equilibrium, it follows by Theorem 1 (i) that $p<p_{m}$, and therefore by Lemma A.4 that

$$
u_{i}\left(a_{m-1}, s_{-i}\right)-u_{i}\left(a_{m}, s_{-i}\right) \geq \frac{1}{N}
$$

for all $s_{-i} \in B_{-i}^{m}$ such that $u_{i}\left(a_{m}, s_{-i}\right)>0$. Moreover, by the definition of the utility function

$$
\frac{1}{m-1} \sum_{n=0}^{m-2} u_{i}\left(a_{n}, s_{-i}\right)-u_{i}\left(a_{m}, s_{-i}\right) \geq-1 \text {. }
$$

Multiply both sides of (A.6) with $\lambda$, and both sides of (A.7) with $1-\lambda$, and add the respective sides to obtain

$$
\begin{aligned}
u_{i}\left(\sigma_{i}^{m}, s_{-i}\right)-u_{i}\left(a_{m}, s_{-i}\right) & \geq \frac{\lambda}{N}+\lambda-1 \\
& =\lambda \frac{1+N}{N}-1>0,
\end{aligned}
$$

with the strict inequality in (A.8) following directly by $\lambda>\frac{N}{1+N}$, which is true by our construction of $\sigma_{i}^{m}$. Hence, we obtain $u_{i}\left(\sigma_{i}^{m}, s_{-i}\right)>u_{i}\left(a_{m}, s_{-i}\right)$.

Combining the previous two cases proves that $a_{m}$ is strictly dominated by $\sigma_{i}^{m}$ in $B_{1}^{m} \times \cdots \times B_{N}^{m}$.

Proof of Lemma 4. The proof is similar to the one of Lemma 3.

(i) Fix some $m \in\{0, \ldots, M-1\}$ and define a strategy profile

$$
\bar{s}_{m}^{\kappa}=(\underbrace{a_{m}, \ldots, a_{m}}_{N-\kappa-1 \text { times }}, \underbrace{a_{m+1}, \ldots, a_{m+1}}_{\kappa \text { times }}) \in S_{-i}
$$

where $\kappa \in\{0, \ldots, N-1\}$. If $\left(a_{m}, \ldots, a_{m}\right)$ is not a Nash equilibrium, $a_{m+1}$ is a best response to $\bar{s}_{m}^{0}$ (by Lemma 2), whereas if $\left(a_{m+1}, \ldots, a_{m+1}\right)$ is a Nash equilibrium, $a_{m+1}$ is trivially a best response to $\bar{s}_{m}^{N-1}$. So, we focus on the case where $\left(a_{m}, \ldots, a_{m}\right)$ is a Nash equilibrium while $\left(a_{m+1}, \ldots, a_{m+1}\right)$ is not. This implies $p>1$. Consider the strictly increasing sequence

$$
\left(t\left(a_{m}, \bar{s}_{m}^{0}\right), t\left(a_{m+1}, \bar{s}_{m}^{0}\right), t\left(a_{m+1}, \bar{s}_{m}^{1}\right), \ldots, t\left(a_{m+1}, \bar{s}_{m}^{N-1}\right)\right)
$$

of the target guesses. Note that the difference 


$$
t\left(a_{m+1}, \bar{s}_{m}^{0}\right)-t\left(a_{m}, \bar{s}_{m}^{0}\right)=t\left(a_{m+1}, \bar{s}_{m}^{\kappa+1}\right)-t\left(a_{m-1}, \bar{s}_{m}^{\kappa}\right)=\frac{p}{N}\left(a_{m+1}-a_{m}\right)
$$

does not depend on $\kappa$. Moreover, since $\left(a_{m}, \ldots, a_{m}\right)$ is a Nash equilibrium,

$$
a_{m} \leq t\left(a_{m}, \bar{s}_{m}^{0}\right)<t\left(a_{m+1}, \bar{s}_{m}^{0}\right) \leq \frac{1}{2}\left(a_{m}+a_{m+1}\right)<a_{m+1} \leq t\left(a_{m+1}, \bar{s}_{m}^{N-1}\right) .
$$

Then we define $\kappa^{*}:=\min \left\{k \in\{1, \ldots, N-1\}: \frac{1}{2}\left(a_{m}+a_{m+1}\right)<t\left(a_{m+1}, \bar{s}_{m}^{\kappa}\right) \leq a_{m+1}\right\}$. By (A.9) and (A.10), we obtain $\frac{p}{N} \leq \frac{1}{2}$, and hence such a $\kappa$ exists. In fact if it holds with equality then $t\left(a_{m+1}, \bar{s}_{m}^{0}\right)=\frac{1}{2}\left(a_{m}+a_{m+1}\right)$, in which case $a_{m+1}$ is a best response to $\bar{a}_{m}^{-i}$, and we are done. Hence, we take $\frac{p}{N}<\frac{1}{2}$. Consider the following cases:

- Let $\kappa^{*} \leq \frac{N-1}{2}$. Let us first prove that

$$
u_{i}\left(a_{m+1}, \bar{s}_{m}^{\kappa^{*}}\right) \geq u_{i}\left(a_{n}, \bar{s}_{m}^{\kappa^{*}}\right)
$$

for all $n \in\{0, \ldots, m-1\}$. Note that

$$
u_{i}\left(a_{m+1}, \bar{s}_{m}^{\kappa^{*}}\right)=\frac{1}{\kappa^{*}+1} \geq \frac{1}{N-\kappa^{*}}=u_{i}\left(a_{m}, \bar{s}_{m}^{\kappa^{*}}\right) .
$$

Moreover, by Lemma A.3 (ii), it follows that (A.11) holds for every $n \in\{0, \ldots, m-1\}$. Now, let us prove it for every $n \in\{m+2, \ldots, M\}$. By construction, $t\left(a_{m+1}, \bar{s}_{m}^{\kappa^{*}}\right) \leq a_{m+1}$. Hence, by $\frac{p}{N} \leq \frac{1}{2}$,

$$
\begin{aligned}
t\left(a_{m+2}, \bar{s}_{m}^{\kappa^{*}}\right) & =t\left(a_{m+1}, \bar{s}_{m}^{\kappa^{*}}\right)+\frac{p}{N}\left(a_{m+2}-a_{m+1}\right) \\
& <a_{m+1}+\frac{1}{2}\left(a_{m+2}-a_{m+1}\right) \\
& =\frac{1}{2}\left(a_{m+1}+a_{m+2}\right),
\end{aligned}
$$

implying that $d_{i}\left(a_{m+2}, \bar{s}_{m}^{\kappa^{*}}\right)>d_{j}\left(a_{m+2}, \bar{s}_{m}^{\kappa^{*}}\right)$, with $j \neq i$ being an opponent of $i$ who chooses $a_{m+1}$ (recall that such $j$ exists, since $\left.\kappa^{*} \geq 1\right)$. Hence, $u_{i}\left(a_{m+1}, \bar{s}_{m}^{\kappa^{*}}\right)>u_{i}\left(a_{m+2}, \bar{s}_{m}^{\kappa^{*}}\right)$, and thus (A.11) follows for all $n \in\{m+2, \ldots, M\}$ by Lemma A.3 (i). Hence $a_{m+1}$ is a best response to $\bar{s}_{m}^{\kappa^{*}}$.

- Let $\kappa^{*}>\frac{N-1}{2}$, i.e., more than half of the opponents choose $a_{m+1}$. Since $\kappa^{*} \in \mathbb{N}$, we have $\kappa^{*} \geq N-\kappa^{*}$. Since additionally $p>1$, we obtain $t\left(a_{m-1}, \underline{s}_{m}^{\kappa^{*}-1}\right)=p \frac{\kappa^{*} a_{m+1}+\left(N-\kappa^{*}\right) a_{m}}{N}>\frac{a_{m}+a_{m+1}}{2}$, a contradiction to the definition of $\kappa^{*}$.

Combining the previous cases completes the proof.

(ii) The proof is almost identical to the one of Lemma A.4. Consider the mixed strategy $\sigma_{i}^{m} \in \Delta\left(A_{i}^{m}\right)$, attaching probability $\lambda \in\left(\frac{N}{1+N}, 1\right)$ to $a_{m+1}$ and equal probability $\frac{1-\lambda}{M-m-1}$ to each $a_{n} \in\left\{a_{m+2}, \ldots, a_{M}\right\}$. Then, consider the following two cases:

- Let $u_{i}\left(a_{m}, s_{-i}\right)=0$. Then, by Lemma A.1, we obtain

$$
u_{i}\left(\sigma_{i}^{m}, s_{-i}\right)=\lambda u_{i}\left(a_{m+1}, s_{-i}\right)+\frac{1-\lambda}{M-m-1} \sum_{n=m+2}^{M} u_{i}\left(a_{n}, s_{-i}\right)>0=u_{i}\left(a_{m}, s_{-i}\right) .
$$

- Let $u_{i}\left(a_{m}, s_{-i}\right)>0$. Since $\left(a_{m}, \ldots, a_{m}\right)$ is not a Nash equilibrium, it follows by Theorem 1 (ii) that $p>p_{m}$, and therefore by Lemma A.5 that

$$
u_{i}\left(a_{m+1}, s_{-i}\right)-u_{i}\left(a_{m}, s_{-i}\right) \geq \frac{1}{N}
$$

for all $s_{-i} \in A_{-i}^{m}$ such that $u_{i}\left(a_{m}, s_{-i}\right)>0$. Moreover, by the definition of the utility function

$$
\frac{1}{M-m-1} \sum_{n=m+2}^{M} u_{i}\left(a_{n}, s_{-i}\right)-u_{i}\left(a_{m}, s_{-i}\right) \geq-1
$$

Multiply (A.12) by $\lambda$ and (A.13) by $1-\lambda$, and add the respective sides to obtain

$$
u_{i}\left(\sigma_{i}^{m}, s_{-i}\right)-u_{i}\left(a_{m}, s_{-i}\right) \geq \frac{\lambda}{N}+\lambda-1>0,
$$

with the strict inequality following from $\lambda>\frac{N}{1+N}$, thus obtaining $u_{i}\left(\sigma_{i}^{m}, s_{-i}\right)>u_{i}\left(a_{m}, s_{-i}\right)$.

Combining the previous two cases proves that $a_{m}$ is strictly dominated by $\sigma_{i}^{m}$ in $A_{1}^{m} \times \cdots \times A_{N}^{m}$. 
Proof of Theorem 2. We begin with sufficiency. Let $\left(a_{m}, \ldots, a_{m}\right)$ and $\left(a_{n}, \ldots, a_{n}\right)$ be two symmetric Nash equilibria (with $m<n)$. For every $i \in I$, take $C_{i}^{m, n}:=A_{i}^{m} \cap B_{i}^{n}=\left\{a_{m}, a_{m+1}, \ldots, a_{n}\right\}$. Then, it suffices to prove that

$$
C_{1}^{m, n} \times \cdots \times C_{N}^{m, n}
$$

is a best response set. Consider the following two cases:

- Let $p \leq 1$. It follows from Lemma 3 (i) that for all $\ell \in\{m, \ldots, n-1\}$ there exists some $s_{-i} \in\left\{s_{-i}^{\prime} \in S_{-i}: s_{j}^{\prime} \in\right.$ $\left\{a_{\ell}, a_{\ell+1}\right\}$ for all $\left.j \neq i\right\} \subseteq C_{-i}^{m, n}$ such that $a_{\ell}$ is a best response to $s_{-i}$. Moreover, by $\left(a_{m}, \ldots, a_{m}\right)$ being a Nash equilibrium, $a_{m}$ is a best response to $\bar{a}_{m}^{-i} \in C_{-i}^{m, n}$.

- Now let $p \geq 1$. It follows from Lemma 4 (i) that for all $\ell \in\{m+1, \ldots, n\}$ there exists some $s_{-i} \in\left\{s_{-i}^{\prime} \in S_{-i}: s_{j}^{\prime} \in\right.$ $\left\{a_{\ell-1}, a_{\ell}\right\}$ for all $\left.j \neq i\right\} \subseteq C_{-i}^{m, n}$ such that $a_{\ell}$ is a best response to $s_{-i}$. Moreover, by $\left(a_{n}, \ldots, a_{n}\right)$ being a Nash equilibrium, $a_{n}$ is a best response to $\bar{a}_{n}^{-i} \in C_{-i}^{m, n}$.

Combining the two cases completes the proof of sufficiency for every $p \in \mathbb{R}_{+}$. Necessity is proven separately for $p \leq 1$ and $p \geq 1$ below.

(i) It follows from Theorem 1 that $\left(a_{0}, \ldots, a_{0}\right)$ is a Nash equilibrium, and therefore also rationalizable. Define $\bar{m}:=$ $\max \left\{n \in\{0, \ldots, M\}: p \geq p_{n}\right\}$. Again by Theorem 1 it follows that $\left(a_{\bar{m}}, \ldots, a_{\bar{m}}\right)$ is a Nash equilibrium. Hence, $a_{m}$ is rationalizable for every $m \in\{0, \ldots, \bar{m}\}$. For $\bar{m}<M$, it remains to prove that no $m \in\{\bar{m}+1, \ldots, M\}$ is rationalizable. We proceed by induction. It follows from Lemma 3 (ii) that for an arbitrary $m \in\{\bar{m}+1, \ldots, M\}$ the strategy $a_{m}$ is strictly dominated in $B_{1}^{m} \times \cdots \times B_{N}^{m}$, and therefore $a_{m} \notin R_{i}^{M-m+1}$. Hence, $a_{m} \notin R_{i}^{*}$, thus completing this part of the proof.

(ii) The proof follows the same steps as Part (i) above. It follows from Theorem 1 that $\left(a_{M}, \ldots, a_{M}\right)$ is rationalizable. Define $\underline{m}:=\min \left\{n \in\{0, \ldots, M\}: p \leq q_{n}\right\}$. Again by Theorem 1 it follows that $\left(a_{m}, \ldots, a_{m}\right)$ is rationalizable for every $m \in$ $\{\underline{m}, \ldots, M\}$. For $\underline{m}>0$, it remains prove that no $m \in\{0, \ldots, \underline{m}-1\}$ is rationalizable. We proceed by induction. It follows from Lemma $4 \overline{(\mathrm{ii})}$ that for an arbitrary $m \in\{0, \ldots, \underline{m}-1\}$ the strategy $a_{m}$ is strictly dominated in $A_{1}^{m} \times \cdots \times A_{N}^{m}$, and therefore $a_{m} \notin R_{i}^{m+1}$. Hence, $a_{m} \notin R_{i}^{*}$, thus completing the proof.

\section{A.4. Proofs of Section 3}

Proof of Corollary 1. When the strategy set is $S_{i}=\{0, \ldots, M\}$, the threshold parameters become $p_{m}=\frac{2 N m-N}{2 N m-2}$ for $m \in$ $\{1, \ldots, M\}$ and $p_{0}=0$. Observe that $p_{m}$ is nondecreasing in $m \in\{0,1, \ldots, M\}$ (resp., strictly increasing if $p<1$ ). Hence, for an arbitrary $p \in[0,1]$, we find $\bar{m}=\max \left\{n \in\{0, \ldots, M\}: p \geq p_{n}\right\}$, noticing that $p \geq p_{m}$ if and only if $m \leq \bar{m}$. But then, it follows from Theorem 2 (ii) that $p \geq p_{m}$ if and only if $m$ is rationalizable.

Proof of Corollary 2. When the strategy set is $S_{i}=\{0, \ldots, M\}$, the threshold parameters become $q_{m}=\frac{2 N m+N}{2 N m+2}$ for $m \in$ $\{0, \ldots, M-1\}$ and $q_{M}=\infty$. Hence, $q_{m}$ is nonincreasing for $m \in\{0, \ldots, M-1\}$, thus implying $q_{M}>q_{0} \geq q_{m}$ for all $m \in$ $\{0, \ldots, M-1\}$.

(i) Take an arbitrary $p \leq \frac{N}{2}=q_{0}$. By Theorem 1 (ii), the profiles $(0, \ldots, 0)$ and $(M, \ldots, M)$ are Nash equilibria. Hence, by Theorem 2 , every $m \in\{0, \ldots, M\}$ is rationalizable.

(ii) Take an arbitrary $p>\frac{N}{2}=q_{0}$, thus implying $p>q_{m}$ for every $m \in\{0, \ldots, M-1\}$. Hence, by Theorem 1 (ii), the only Nash equilibrium is $(M, \ldots, M)$. Thus, by Theorem 2 (ii), the only rationalizable strategy is $M$.

\section{A.5. Proofs of Section 5}

Proof of Proposition 1. We know that in general $S_{i}^{*} \subseteq R_{i}^{*}$. Hence, it suffices to show that $R_{i}^{*} \subseteq S_{i}^{*}$. Take $p \leq 1$. Then, by Theorem 2 (i), we obtain $R_{i}^{*}=\left\{a_{0}, \ldots, a_{\bar{m}}\right\}$. Note that $R_{1}^{*} \times \cdots \times R_{i}^{*}$ is a independent best response set. Indeed, $a_{\bar{m}}$ is a best response to the (independent) belief that puts probability 1 to $\bar{a}_{\bar{m}}^{-i}$, and for every $m \in\{0, \ldots, \bar{m}\}$, the pure strategy $a_{m}$ is a best response to an (independent) belief that puts probability 1 to some $s_{-i} \in\left\{s_{-i}^{\prime} \in S_{-i}: s_{j} \in\left\{a_{m}, a_{m+1}\right\}\right.$ for all $\left.j \neq i\right\} \subseteq R_{-i}^{*}$ by Lemma 3 (ii). The proof is similar for $p \geq 1$. In particular, we obtain $R_{i}^{*}=\left\{a_{m}, \ldots, a_{M}\right\}$. Then, $a_{M}$ is a rational for the (independent) belief that puts probability 1 to $\bar{a}_{M}^{-i}$, and for every $m \in\{\underline{m}-1, \ldots, M\}$, the pure strategy $a_{m}$ is a best response to an (independent) belief that puts probability 1 to some $s_{-i} \in\left\{s_{-i}^{\prime} \in S_{-i}: s_{j} \in\left\{a_{m}, a_{m-1}\right\}\right.$ for all $\left.j \neq i\right\} \subseteq R_{-i}^{*}$ by Lemma 4 (ii).

Proof of Proposition 2. (i) Note that in any game $B_{i}^{m}$, by Lemma 3 (i), only strategy $a_{m}$ can be dominated. By our characterization of $R_{i}^{*}$, strategy $a_{m}$ is dominated if and only if $m>\bar{m}$. Thus, strategy $a_{m}$ is eliminated in round $k+1$ if and only if $m=M-k>\bar{m}$, i.e., formally, $R_{i}^{k+1}=R_{i}^{k} \backslash\left\{a_{M-k}\right\}$ in this case.

(ii) The proof is analogous to Part (i). In any game $A_{i}^{m}$, by Lemma 4 (i), only strategy $a_{m}$ can be dominated. By our characterization of $R_{i}^{*}$, strategy $a_{m}$ is dominated if and only if $m<\underline{m}$. Thus, strategy $a_{m}$ is eliminated in round $k+1$ if and only if $m=k<\underline{m}$, i.e., $R_{i}^{k+1}=R_{i}^{k} \backslash\left\{a_{k}\right\}$. 


\section{References}

Arieli, I., 2010. Rationalizability in continuous games. J. Math. Econ. 46, 912-924.

Bernheim, D., 1984. Rationalizable strategic behavior. Econometrica 52, 1007-1028.

Bosch-Doménech, A., Montalvo, J.G., Nagel, R., Satorra, A., 2002. One, two, (three), infinity, ...: newspaper and lab beauty-contest experiments. Amer. Econ. Rev. 92, 1678-1701.

Brandenburger, A., Dekel, E., 1987. Rationalizability and correlated equilibria. Econometrica 55, 1391-1402.

Camerer, C., Ho, T.H., Chong, J.K., 2004. A cognitive hierarchy model of games. Quart. J. Econ. 119, 861-898.

Ho, T.H., Camerer, C., Weigelt, K., 1998. Iterated dominance and iterated best response in experimental “p-beauty contests". Amer. Econ. Rev. 88, 947-969. Ledoux, A., 1981. Concours résultats complets. Les victimes se sont plu á jouer le 14 d'atout. Jeux \& Stratégie 2, 10-11.

López, R., 2001. On p-Beauty Contest Integer Games. Universitat Pompeu Fabra Working Paper.

Moulin, H., 1986. Game Theory for Social Sciences. New York University Press.

Nagel, R., 1995. Unraveling in guessing games: an experimental study. Amer. Econ. Rev. 85, 1313-1326.

Pearce, D.G., 1984. Rationalizable strategic behavior and the problem of perfection. Econometrica 52, 1029-1050.

Tan, T., Werlang, S., 1988. The Bayesian foundations of solution concepts of games. J. Econ. Theory 45, 370-391. 